\title{
NEW LOWER SOLUTION BOUNDS FOR THE CONTINUOUS ALGEBRAIC RICCATI EQUATION*
}

\author{
JUAN ZHANG ${ }^{\dagger}$ AND JIANZHOU LIU $^{\dagger}$
}

Abstract. In this paper, by constructing the equivalent form of the continuous algebraic Riccati equation (CARE) and applying some matrix inequalities, a new lower bounds solution of the CARE is proposed. Finally, corresponding numerical examples are provided to illustrate the effectiveness of the results.

Key words. Eigenvalue, Continuous algebraic Riccati equation, Matrix bound.

AMS subject classifications. 15A24.

1. Introduction. It is known to us that the algebraic Riccati equations, discrete matrix equations, and matrix functions are of great importance in many areas such as optimal control, filter design and stability analysis, and so on (see [7], [8], [11], [13], [19], [20], [26]). Consider the following linear system (see [20]):

$$
\left\{\begin{array}{l}
\dot{x}(t)=A x(t)+B u(t) \\
x(0)=x_{0}
\end{array}\right.
$$

where $A \in \mathbb{R}^{n \times n}, B \in \mathbb{R}^{n \times m}, x_{0} \in \mathbb{R}^{n}$ is the initial state. And the state feedback control and the performance index of the system (1.1), respectively, are

$$
u(t)=-K x(t), \quad K=B^{T} P,
$$

and

$$
J=\int_{0}^{\infty}\left(x^{T} Q x+u^{T} u\right) d t
$$

where $Q \in \mathbb{R}^{n \times n}$ is symmetric positive definite, $P$ is the symmetric positive semidefinite solution of the continuous algebraic Riccati equation (CARE)

$$
A^{T} P+P A-P B B^{T} P=-Q .
$$

*Received by the editors on January 15, 2010. Accepted for publication on February 13, 2011. Handling Editor: Daniel Szyld.

${ }^{\dagger}$ Department of Mathematics and Computational Science, Xiangtan University, Xiangtan, Hunan 411105, China (liujz@xtu.edu.cn). Supported in part by Natural Science Foundation of China (10971176) and the Key Project of Hunan Provincial Natural Science Foundation of China (10JJ2002). 
To guarantee the existence of the stabilizing solution of the CARE (1.2), it shall be assumed that the pair $(A, B)$ is stabilizable, and the pair $\left(Q^{\frac{1}{2}}, A\right)$ is observable.

What is more, by [11] and [25], it can be seen that in the optimal regulator problem, the optimal cost can be written as

$$
J^{*}=x_{0}^{T} P x_{0}
$$

where $x_{0}$ is the initial state of the system (1.1) and $P$ is the symmetric positive definite solution of the CARE (1.2). An interpretation of $\operatorname{tr}(P)$ is that $\operatorname{tr}(P) / n$ is the average value of the cost $J^{*}$ as $x_{0}$ varies over the surface of a unit sphere.

Therefore, considering its important applications during the past two and three decades, many researchers start to studying the solution of the CARE (1.2). While in practice, it is hard to solve the CARE, and there is no general method unless the system matrices are special and there are some methods and algorithms to solve (1.2) (see [9] and [23]). However, the solution of this equation can be time-consuming and computationally difficult, particularly as the dimensions of the system matrices increase. Meantime, the solution bounds can also be used as the approximations of the exact solution for the CARE (1.2) (Barnett and Storey 1970 [1], Patel and Toda 1984 [22], Mori and Derese 1984 [18], Kwon, Moon, and Ahn 1996 [15]). Hence, there are many papers for deriving the bounds of the solution for the CARE (Kwon and Pearson 1977 [16], Patel and Toda 1978 [21], Yasuda and Hirai 1979 [27], Karanam 1983 [10], Kwon, Youn, and Bien 1985 [14], Wang, Kuo, and Hsu 1986 [25], Saniuk and Rhodes 1987 [24], Kwon, Moon, and Ahn 1996 [15], Lee, 1997 [17], Czornik and Nawrat, 2000 [5], Choi and Kuc, 2002 [4], Davies, Shi, and Wiltshire 2007 [6], Chen and Lee, 2009 [3]). The previous results during 1974-1994 have been summarized in Kwon et al. [15] (1996). Of these bounds, the matrix bounds are the most general and desirable as they can offer all other types of bounds. In this paper, by constructing the equivalent form of the CARE, utilizing some matrix inequalities, we develop new lower matrix bounds. Finally, we give some numerical examples to illustrate the effectiveness of the derived results.

Throughout this paper, let $\mathbb{R}^{n \times m}$ and $\mathbb{R}^{n}$ denote the set of $n \times m$ real matrices and $n$-dimensional column vector. If $X \in \mathbb{R}^{n \times n}$ is an arbitrary symmetric matrix, we assume that the eigenvalues of $X$ are arranged so that $\lambda_{1}(X) \geq \lambda_{2}(X) \geq \cdots \geq \lambda_{n}(X)$. For $X, Y \in \mathbb{R}^{n \times n}$, let $\operatorname{tr}(X), X^{T}, X^{-1}, \operatorname{det}(X),\|X\|$ denote the trace, the transpose, the inverse, the determinant and the spectral norm of $X$, respectively. The inequality $X>(\geq) 0$ means that $X$ is a symmetric positive (semi-) definite matrix and $X>(\geq) Y$ means that $X-Y$ is a symmetric positive (semi-) definite matrix. The identity matrix with appropriate dimensions is represented by $I$.

The following lemmas are used to prove the main results. 
Lemma 1.1. [2] For any symmetric matrix $X \in \mathbb{R}^{n \times n}$, the following inequality holds:

$$
\lambda_{n}(X) I \leq X \leq \lambda_{1}(X) I
$$

LEMma 1.2. [27] The positive semi-definite solution $P$ to the CARE (1.2) has the following lower bound on its minimum eigenvalue:

$$
\lambda_{n}(P) \geq \frac{\lambda_{n}\left(\bar{A} Q^{-1}\right)+\sqrt{\left[\lambda_{n}\left(\bar{A} Q^{-1}\right)\right]^{2}+\lambda_{1}\left(B B^{T} Q^{-1}\right)}}{\lambda_{1}\left(B B^{T} Q^{-1}\right)} \equiv \kappa,
$$

with $\bar{A}=\frac{A+A^{T}}{2}$.

LEMMA 1.3. [12] The following matrix inequality

$$
\left(\begin{array}{ll}
W & S \\
S^{T} & V
\end{array}\right)>0
$$

where $W=W^{T}$ and $V=V^{T}$, is equivalent to either

$$
V>0, \quad W-S V^{-1} S^{T}>0
$$

or

$$
W>0, \quad V-S^{T} W^{-1} S>0
$$

2. New lower solution bounds for the CARE. In this section, we present new lower matrix bounds of the solution for the continuous algebraic Riccati equation.

TheOrem 2.1. The solution $P$ to the CARE (1.2) satisfies

$$
P \geq\left\{\varepsilon Q-\varepsilon^{2}(V+I)^{T}\left(I-\varepsilon B B^{T}\right)^{-1}(V+I)+\varepsilon^{2} \alpha^{2} I\right\}^{\frac{1}{2}}+\varepsilon \alpha I \equiv P_{l 1},
$$

where the positive constant $\alpha$ is chosen so that

$$
A+A^{T}<2 \alpha I
$$

the matrix $V$ is defined by

$$
V \equiv A-\alpha I-I
$$

and $\varepsilon$ is any positive constant such that

$$
0<\varepsilon<\min \left\{b,\left\|B B^{T}+(V+I) Q^{-1}(V+I)^{T}\right\|^{-1}\right\},
$$


where $b \equiv \frac{\kappa}{\alpha}, \kappa$ is defined by (1.3).

Proof. The CARE (1.2) can be rewritten as

$$
P(A-\alpha I)+(A-\alpha I)^{T} P+2 \alpha P+Q=P B B^{T} P
$$

where $\alpha$ is a positive constant. Via the definition of $V$ from (2.3), then

$$
P(V+I)+(V+I)^{T} P+2 \alpha P+Q=P B B^{T} P .
$$

Adding and subtracting $\frac{1}{\varepsilon} P P+(V+I)^{T}\left(\frac{1}{\varepsilon} I-B B^{T}\right)^{-1}(V+I)$ from $(2.5)$ leads to

$$
\begin{aligned}
& {\left[P+\left(\frac{1}{\varepsilon} I-B B^{T}\right)^{-1}(V+I)\right]^{T}\left(\frac{1}{\varepsilon} I-B B^{T}\right)\left[P+\left(\frac{1}{\varepsilon} I-B B^{T}\right)^{-1}(V+I)\right]} \\
& -\frac{1}{\varepsilon} P P-(V+I)^{T}\left(\frac{1}{\varepsilon} I-B B^{T}\right)^{-1}(V+I)+2 \alpha P+Q=0 .
\end{aligned}
$$

Hence,

$$
\text { (2.6) } \begin{aligned}
& \frac{1}{\varepsilon} P P+(V+I)^{T}\left(\frac{1}{\varepsilon} I-B B^{T}\right)^{-1}(V+I)-2 \alpha P-Q \\
= & {\left[P+\left(\frac{1}{\varepsilon} I-B B^{T}\right)^{-1}(V+I)\right]^{T}\left(\frac{1}{\varepsilon} I-B B^{T}\right)\left[P+\left(\frac{1}{\varepsilon} I-B B^{T}\right)^{-1}(V+I)\right] \geq 0 . }
\end{aligned}
$$

As $Q>0$ and $\varepsilon$ satisfies $(2.4)$, then

$$
I-\varepsilon B B^{T}-\varepsilon(V+I) Q^{-1}(V+I)^{T}>0, \quad \varepsilon Q>0 .
$$

Using Lemma 1.3, we can see that the above inequalities are satisfied if and only if

$$
\left(\begin{array}{cc}
\varepsilon Q & \varepsilon(V+I)^{T} \\
\varepsilon(V+I) & I-\varepsilon B B^{T}
\end{array}\right)>0
$$

which is equivalent to

$$
\varepsilon Q-\varepsilon^{2}(V+I)^{T}\left(I-\varepsilon B B^{T}\right)^{-1}(V+I)>0, \quad I-\varepsilon B B^{T}>0 .
$$

Then from (2.6), it is easy to see that

$$
P P-2 \varepsilon \alpha P \geq \varepsilon Q-\varepsilon^{2}(V+I)^{T}\left(I-\varepsilon B B^{T}\right)^{-1}(V+I) .
$$

Considering (2.7), the above inequality is equivalent to

$$
(P-\varepsilon \alpha I)^{2} \geq \varepsilon Q-\varepsilon^{2}(V+I)^{T}\left(I-\varepsilon B B^{T}\right)^{-1}(V+I)+\varepsilon^{2} \alpha^{2} I>0 .
$$


On the other hand, if $\varepsilon$ satisfies (2.4), then

$$
\varepsilon<\frac{\kappa}{\alpha}
$$

Applying (1.3) and Lemma 1.1 to (2.9) satisfies

$$
\varepsilon I<\frac{\kappa}{\alpha} I<\frac{\lambda_{n}(P)}{\alpha} I<\frac{P}{\alpha} .
$$

Then $P-\varepsilon \alpha I>0$. Consequently, (2.8) changes to

$$
P-\varepsilon \alpha I \geq\left\{\varepsilon Q-\varepsilon^{2}(V+I)^{T}\left(I-\varepsilon B B^{T}\right)^{-1}(V+I)+\varepsilon^{2} \alpha^{2} I\right\}^{\frac{1}{2}},
$$

which implies that

$$
P \geq\left\{\varepsilon Q-\varepsilon^{2}(V+I)^{T}\left(I-\varepsilon B B^{T}\right)^{-1}(V+I)+\varepsilon^{2} \alpha^{2} I\right\}^{\frac{1}{2}}+\varepsilon \alpha I .
$$

This completes the proof.

By using the above Theorem 2.1, we can derive the following result immediately.

Corollary 2.2. The positive semi-definite solution $P$ to the CARE (1.2) satisfies the following lower eigenvalue bounds for the positive constant $\alpha$ satisfying (2.2) and any $\varepsilon$ satisfying (2.4):

$$
\begin{aligned}
& \lambda_{i}(P) \geq \max _{\varepsilon} \lambda_{i}\left(P_{l 1}\right) \equiv P_{i l 1}^{*} \\
& \geq \lambda_{i}\left(P_{l 1}\right) \\
& \operatorname{tr}(P) \geq \sum_{i=1}^{n} P_{i l 1}^{*} \geq \max _{\varepsilon} \operatorname{tr}\left(P_{l 1}\right) \\
& \geq \operatorname{tr}\left(P_{l 1}\right), \\
& \operatorname{det}(P) \geq \prod_{i=1}^{n} P_{i l 1}^{*} \geq \max _{\varepsilon} \operatorname{det}\left(P_{l 1}\right) \\
& \geq \operatorname{det}\left(P_{l 1}\right) .
\end{aligned}
$$

REMARK 2.3. Since $I$ is a positive definite matrix of full rank, there will exist a positive constant $\alpha$ such that condition (2.2) is satisfied. Also, since $\kappa$ is a lower 
bound for $\lambda_{n}(P)$, there will always exist values of $\kappa, b$ such that the conditions (1.3), (2.4) are fulfilled. Hence, the lower bound $P_{l 1}$ is always calculated if the solution of the CARE (1.2) exists.

Following the above results, we now obtain a different lower matrix bound as follows for the CARE (1.2).

THEOREM 2.4. The solution $P$ to the CARE (1.2) satisfies $P \geq\left\{\varepsilon^{*} Q-\left(\varepsilon^{*}\right)^{2}(V+2 I)^{T}\left(I-\varepsilon^{*} B B^{T}\right)^{-1}(V+2 I)+\left(\varepsilon^{*}\right)^{2}(\alpha-1)^{2} I\right\}^{\frac{1}{2}}+\varepsilon^{*}(\alpha-1) I \equiv P_{l 2}$,

where the positive constant $\alpha>1$ is chosen so as to satisfy the condition (2.2), $V$ is defined by (2.3), and $\varepsilon^{*}$ is any positive constant such that

$$
0<\varepsilon^{*}<\min \left\{c,\left\|B B^{T}+(V+2 I) Q^{-1}(V+2 I)^{T}\right\|^{-1}\right\},
$$

where $c \equiv \frac{\kappa}{\alpha-1}, \kappa$ is defined by (1.3).

Proof. Using the definition of $V$ from (2.3), (2.5) can be rewritten as

$$
P V+V^{T} P+2 P+2 \alpha P+Q=P B B^{T} P .
$$

Adding $2 P$ to both sides of (2.12) gives

$$
P(V+2 I)+(V+2 I)^{T} P+2 P(\alpha-1)+Q=P B B^{T} P .
$$

Adding and subtracting $\frac{1}{\varepsilon^{*}} P P+(V+2 I)^{T}\left(\frac{1}{\varepsilon^{*}} I-B B^{T}\right)^{-1}(V+2 I)$ from (2.13) leads to

$$
\begin{aligned}
& {\left[P+\left(\frac{1}{\varepsilon^{*}} I-B B^{T}\right)^{-1}(V+2 I)\right]^{T}\left(\frac{1}{\varepsilon^{*}} I-B B^{T}\right)\left[P+\left(\frac{1}{\varepsilon^{*}} I-B B^{T}\right)^{-1}(V+2 I)\right]} \\
& -\frac{1}{\varepsilon^{*}} P P-(V+2 I)^{T}\left(\frac{1}{\varepsilon^{*}} I-B B^{T}\right)^{-1}(V+2 I)+2 P(\alpha-1)+Q=0 .
\end{aligned}
$$

Thus,

(2.14) $\frac{1}{\varepsilon^{*}} P P+(V+2 I)^{T}\left(\frac{1}{\varepsilon^{*}} I-B B^{T}\right)^{-1}(V+2 I)-2 P(\alpha-1)-Q$

$$
=\left[P+\left(\frac{1}{\varepsilon^{*}} I-B B^{T}\right)^{-1}(V+2 I)\right]^{T}\left(\frac{1}{\varepsilon^{*}} I-B B^{T}\right)\left[P+\left(\frac{1}{\varepsilon^{*}} I-B B^{T}\right)^{-1}(V+2 I)\right] \geq 0 .
$$

As $Q>0$ and $\varepsilon^{*}$ satisfies $(2.11)$, then

$$
I-\varepsilon^{*} B B^{T}-\varepsilon^{*}(V+2 I) Q^{-1}(V+2 I)^{T}>0, \quad \varepsilon Q>0 .
$$


Using Lemma 1.3, we can see that the above inequalities are satisfied if and only if

$$
\left(\begin{array}{cc}
\varepsilon^{*} Q & \varepsilon^{*}(V+2 I)^{T} \\
\varepsilon^{*}(V+2 I) & I-\varepsilon^{*} B B^{T}
\end{array}\right)>0,
$$

which is equivalent to

(2.15) $\varepsilon^{*} Q-\left(\varepsilon^{*}\right)^{2}(V+2 I)^{T}\left(I-\varepsilon^{*} B B^{T}\right)^{-1}(V+2 I)>0, \quad I-\varepsilon^{*} B B^{T}>0$.

Then from (2.14), it is simple to see that

$$
P P-2 \varepsilon^{*} P(\alpha-1) \geq \varepsilon^{*} Q-\left(\varepsilon^{*}\right)^{2}(V+2 I)^{T}\left(I-\varepsilon^{*} B B^{T}\right)^{-1}(V+2 I) .
$$

Considering (2.15), the above inequality is equivalent to

$$
\begin{gathered}
{\left[P-\varepsilon^{*}(\alpha-1) I\right]^{2}} \\
\geq \varepsilon^{*} Q-\left(\varepsilon^{*}\right)^{2}(V+2 I)^{T}\left(I-\varepsilon^{*} B B^{T}\right)^{-1}(V+2 I)+\left(\varepsilon^{*}\right)^{2}(\alpha-1)^{2} I>0 .
\end{gathered}
$$

On the other hand, if $\varepsilon^{*}$ satisfies (2.11) and $\alpha>1$, then

$$
\varepsilon^{*}<\frac{\kappa}{\alpha-1} \text {. }
$$

Applying (1.3) and Lemma 1.1 to (2.17) yields

$$
\varepsilon^{*} I<\frac{\kappa}{\alpha-1} I<\frac{\lambda_{n}(P)}{\alpha-1} I<\frac{P}{\alpha-1} .
$$

Then $P-\varepsilon^{*}(\alpha-1) I>0$. Hence, (2.16) changes to

$P-\varepsilon^{*}(\alpha-1) I \geq\left\{\varepsilon^{*} Q-\left(\varepsilon^{*}\right)^{2}(V+2 I)^{T}\left(I-\varepsilon^{*} B B^{T}\right)^{-1}(V+2 I)+\left(\varepsilon^{*}\right)^{2}(\alpha-1)^{2} I\right\}^{\frac{1}{2}}$,

which means that

$P \geq\left\{\varepsilon^{*} Q-\left(\varepsilon^{*}\right)^{2}(V+2 I)^{T}\left(I-\varepsilon^{*} B B^{T}\right)^{-1}(V+2 I)+\left(\varepsilon^{*}\right)^{2}(\alpha-1)^{2} I\right\}^{\frac{1}{2}}+\varepsilon^{*}(\alpha-1) I$.

This completes the proof.

By using the above Theorem 2.4, we can derive the following result immediately.

Corollary 2.5. The positive semi-definite solution $P$ to the CARE (1.2) satisfies the following lower eigenvalue bounds for the positive constant $\alpha>1$ satisfying (2.2) and any $\varepsilon^{*}$ satisfying (2.11):

$$
\begin{aligned}
\lambda_{i}(P) & \geq \max _{\varepsilon} \lambda_{i}\left(P_{l 2}\right) \equiv P_{i l 2}^{*} \\
& \geq \lambda_{i}\left(P_{l 2}\right),
\end{aligned}
$$




$$
\begin{aligned}
\operatorname{tr}(P) & \geq \sum_{i=1}^{n} P_{i l 2}^{*} \geq \max _{\varepsilon} \operatorname{tr}\left(P_{l 2}\right) \\
& \geq \operatorname{tr}\left(P_{l 2}\right) \\
\operatorname{det}(P) & \geq \prod_{i=1}^{n} P_{i l 2}^{*} \geq \max _{\varepsilon} \operatorname{det}\left(P_{l 2}\right) \\
& \geq \operatorname{det}\left(P_{l 2}\right) .
\end{aligned}
$$

REMARK 2.6. As in Remark 2.3, the lower bound $P_{l 2}$ is always calculated if the solution of the CARE (1.2) exists.

REMARK 2.7. For one thing, Theorem 2.1 and Theorem 2.4 are two different lower bounds of the solution of the CARE (1.2). It is difficult to compare the sharpness of them, and we will present two examples in Section 3 to illustrate this. On the other hand, from the literature, we know that lower matrix bounds for the solution of the CARE (1.2) have been presented in Kwon and Pearson (1977) [16], Lee (1997) [17], Choi and Kuc (2002) [4], Davies, Shi, and Wiltshire (2007) [6], and Chen and Lee (2009) [3]. As Chen and Lee (2009) [3] pointed out, to give a general comparison between any parallel lower bounds for the same measure is hard. Hence, it is also hard to compare the sharpness of our lower bounds Theorem 2.1 and Theorem 2.4 to these parallel results. Further, in Section 3, we shall give two examples to show that our lower bounds are tighter than the majority of those parallel results for some cases.

3. Numerical examples. In this section, we present numerical examples to illustrate the effectiveness of the main results.

ExAmple 3.1. [6] Consider CARE (1.2) with

$$
A=\left(\begin{array}{cc}
0.5 & 0 \\
1 & -2.5
\end{array}\right), \quad B=\left(\begin{array}{ll}
2 & 0 \\
0 & 0
\end{array}\right), \quad Q=\left(\begin{array}{ll}
1 & 0 \\
0 & 3
\end{array}\right) .
$$

Obviously, the pair $(A, B)$ is stabilizable, and the pair $\left(Q^{\frac{1}{2}}, A\right)$ is observable.

Using the method of Kwon and Pearson (1977) [16], we obtain

$$
P \geq G=\left(\begin{array}{cc}
0.1651 & 0 \\
0 & 0.1651
\end{array}\right)
$$


With $\alpha^{\prime}=5$, using Theorem 4 of Lee (1997) [17], we have

$$
P \geq E=\left(\begin{array}{ll}
0.2887 & 0.2357 \\
0.2357 & 0.4410
\end{array}\right)
$$

Choosing $\varepsilon^{\prime}=\frac{1}{2}\left\|B B^{T}+A Q^{-1} A^{T}\right\|^{-1}=0.1127$, using Theorem 4 of Choi and Kuc (2002) [4], we get

$$
P \geq \Psi=\left(\begin{array}{ll}
0.3070 & 0.1783 \\
0.1783 & 0.5087
\end{array}\right)
$$

With $\eta=0.7974$ and $\alpha=1.5$ as [6], the lower matrix bound found by Theorem 2.1 of Davies et al. (2007) is

$$
P \geq P_{l 1}^{*}=\left(\begin{array}{ll}
0.3659 & 0.0484 \\
0.0484 & 0.2427
\end{array}\right)
$$

Using Theorem 2.1 of Chen and Lee (2009) [3], we have

$$
P \geq \Phi=\left(\begin{array}{ll}
0.2826 & 0.1429 \\
0.1429 & 0.2776
\end{array}\right)
$$

With $\varepsilon^{*}=0.1$, the lower matrix bound found by Theorem $2.4(2.10)$ is

$$
P \geq P_{l 2}=\left(\begin{array}{ll}
0.3541 & 0.1732 \\
0.1732 & 0.5110
\end{array}\right)
$$

With $\varepsilon=0.1$, the lower matrix bound found by Theorem $2.1(2.1)$ is

$$
P \geq P_{l 1}=\left(\begin{array}{cc}
0.4596 & 0.2 \\
0.2 & 0.5531
\end{array}\right)
$$

By computation, it is obvious that

$P_{l 1}-G \geq 0, \quad P_{l 1}-E \geq 0, \quad P_{l 1}-\Psi \geq 0, \quad P_{l 1}-P_{l 1}^{*} \geq 0, \quad P_{l 1}-\Phi \geq 0, \quad P_{l 1}-P_{l 2} \geq 0$, i.e.,

$$
P_{l 1} \geq G, \quad P_{l 1} \geq E, \quad P_{l 1} \geq \Psi, \quad P_{l 1} \geq P_{l 1}^{*}, \quad P_{l 1} \geq \Phi, \quad P_{l 1} \geq P_{l 2}
$$

which implies that our lower bound $P_{l 1}$ is tighter than the parallel results for this case. 
Example 3.2. Consider the CARE (1.2) with

$$
A=\left(\begin{array}{cc}
-1 & 1 \\
0 & 2
\end{array}\right), \quad B=\left(\begin{array}{l}
1 \\
0
\end{array}\right), \quad Q=\left(\begin{array}{ll}
1 & 0 \\
0 & 1
\end{array}\right)
$$

Obviously, the pair $(A, B)$ is stabilizable, and the pair $\left(Q^{\frac{1}{2}}, A\right)$ is observable.

Using the method of Kwon and Pearson (1977) [16], we obtain

$$
P \geq G=\left(\begin{array}{cc}
0.2090 & 0 \\
0 & 0.2090
\end{array}\right)
$$

Choosing $\alpha^{\prime}=10$, using Theorem 4 of Lee (1997) [17], we have

$$
P \geq E=\left(\begin{array}{ll}
0.2860 & 0.0953 \\
0.0953 & 0.2132
\end{array}\right)
$$

With $\varepsilon^{\prime}=\frac{1}{2}\left\|B B^{T}+A Q^{-1} A^{T}\right\|^{-1}=0.0899$, using Theorem 4 of Choi and Kuc (2002) [4], we obtain

$$
P \geq \Psi=\left(\begin{array}{ll}
0.2846 & 0.0942 \\
0.0942 & 0.2207
\end{array}\right)
$$

Choosing $\eta=0.7974$ and $\alpha=10$, the lower matrix bound found by Theorem 2.2 in [6] of Davies et al. (2007) is

$$
P \geq P_{l 2}^{*}=\left(\begin{array}{ll}
0.2459 & 0.0052 \\
0.0052 & 0.2879
\end{array}\right)
$$

Using Theorem 2.1 of Chen and Lee (2009) [3], we have

$$
P \geq \Phi=\left(\begin{array}{ll}
0.2424 & 0.0420 \\
0.0420 & 0.0846
\end{array}\right)
$$

With $\varepsilon=0.008$, the lower matrix bound found by Theorem $2.1(2.1)$ is

$$
P \geq P_{l 1}=\left(\begin{array}{ll}
0.1612 & 0.0266 \\
0.0266 & 0.1812
\end{array}\right)
$$

With $\varepsilon^{*}=0.04$, the lower matrix bound found by Theorem $2.4(2.10)$ is

$$
P \geq P_{l 2}=\left(\begin{array}{ll}
0.4142 & 0.1291 \\
0.1291 & 0.6592
\end{array}\right)
$$


By computation, it is obvious that

$P_{l 2}-G \geq 0, \quad P_{l 2}-E \geq 0, \quad P_{l 2}-\Psi \geq 0, \quad P_{l 2}-P_{l 2}^{*} \geq 0, \quad P_{l 2}-\Phi \geq 0, \quad P_{l 2}-P_{l 1} \geq 0$, i.e.,

$$
P_{l 2} \geq G, \quad P_{l 2} \geq E, \quad P_{l 2} \geq \Psi, \quad P_{l 2} \geq P_{l 2}^{*}, \quad P_{l 2} \geq \Phi, \quad P_{l 2} \geq P_{l 1},
$$

which implies that our lower bound $P_{l 2}$ is tighter than the parallel results for this case.

4. Conclusions. New lower bounds of the solution for the CARE have been provided via the construction of an equivalent form for the CARE and the use of some matrix inequalities. Two examples show that the lower bounds are better than the majority of those parallel results for some cases.

Acknowledgment. The authors would like to thank Professor Daniel Szyld and the referees for their constructive suggestions and helpful comments which greatly improved this paper.

\section{REFERENCES}

[1] S. Barnett and C. Storey. Matrix Methods in Stability Theory. Barnes and Noble Inc., New York, 1970.

[2] D.S. Bernstein. Matrix Mathematics: Theory, Facts, and Formulas With Application to Linear Systems Theory. Princeton University Press, Princeton, NJ, 2005.

[3] C.-Y. Chen and C.-H. Lee. Explicit matrix bounds of the solution for the continuous Riccati equation. ICIC Express Letters, 3:147-152, 2009.

[4] H.H. Choi and T.-Y. Kuc. Lower matrix bounds for the continuous algebraic Riccati and Lyapunov matrix equations. Automatica J. IFAC, 38:1147-1152, 2002.

[5] A. Czornik and A. Nawrat. On the bounds on the solutions of the algebraic Lyapunov and Riccati equations. Arch. Control Sci., 10:197-244, 2000.

[6] R. Davies, P. Shi, and R. Wiltshire. New lower solution bounds of the continuous algebraic Riccati matrix equation. Linear Algebra Appl., 427:242-255, 2007.

[7] R. Davies, P. Shi, and R. Wiltshire. New upper solution bounds for perturbed continuous algebraic Riccati equations applied to automatic control. Chaos Solitons Fractals, 32:487495, 2007.

[8] V. Dragan and T. Morozan. Discrete-time Riccati type equations and the tracking problem. ICIC Express Letters, 2:109-116, 2008.

[9] V.I. Hasanov and S.M. El-Sayed. On the positive definite solutions of the nonlinear matrix equation $X+A^{*} X^{-\delta} A=Q$. Linear Algebra Appl., 412:154-160, 2006.

[10] V.R. Karanam. A note on eigenvalue bounds in algebraic Riccati equation. IEEE Trans. Automat. Contr., 28:109-111, 1983.

[11] D.L. Kleinman and M. Athans. The design of suboptimal linear time-varying systems. IEEE Trans. Automatic Control, 13:150-159, 1968.

[12] E. Kreindler and A. Jameson. Conditions for nonnegativeness of partioned matrices. IEEE Trans. Automat. Contr., 17:147-148, 1972. 
[13] K. Kwakernaak and R. Sivan. Linear Optimal Control Systems. John Wiley \& Sons, New York, 1972.

[14] B.H. Kwon, M.J. Youn, and Z. Bien. On bounds of the Riccati and Lyapunov matrix equations. IEEE Trans. Automat. Contr., 30:1134-1135, 1985.

[15] W.H. Kwon, Y.S. Moon, and S.C. Ahn. Bounds on solutions of algebraic Riccati and Lyapunov equations: a survey and new results. Internat. J. Control, 64:377-389, 1996.

[16] W.H. Kwon and A.E. Pearson. A note on the algebraic matrix Riccati equation IEEE Trans. Automat. Control, 22:143-144, 1977.

[17] C.-H. Lee. On the upper and lower bounds of the solution for the continuous Riccati matrix equation. Internat. J. Control, 66:105-118, 1997.

[18] T. Mori and I.A. Derese. A brief summary of the bounds on the solution of the algebraic matrix equations in control theory. Internat. J. Control, 39:247-256, 1984.

[19] K. Ogata. Modern Control Engineering, third edition. Prentice Hall, Upper Saddle River, NJ, 1997.

[20] M.-L. Ni. Existence condition on solutions to the algebraic Riccati equation. Acta Automat. Sinica, 34:85-87, 2008.

[21] R.V. Patel and M. Toda. On norm bounds for algebraic Riccati and Lyapunov equations. IEEE Trans. Automat. Contr., 23:87-88, 1978.

[22] R.V. Patel and M. Toda. Bounds on performance of nonstationary continuous-time filters under modelling uncertainty. Automatica J. IFAC, 20:117-120, 1984.

[23] Z. Peng, S.M. El-Sayed, and X. Zhang. Iterative methods for the extremal positive definite solution of the matrix equation $X+A^{*} X^{-\alpha} A=Q$. J. Comput. Appl. Math., 200:520-527, 2007.

[24] J.M. Saniuk and I.B. Rhodes. A matrix inequality associated with bounds on solutions of algebraic Riccati and Lyapunov equations. IEEE Trans. Automat. Contr., 32:739-740, 1987.

[25] S.D. Wang, T.S. Kuo, and C.F. Hsu. The bounds on the solution of the algebraic Riccati and Lyapunov equation. IEEE Trans. Automat. Control, 31:654-656, 1986.

[26] X.-T. Wang. Numerical solution of optimal control for scaled systems by hybrid functions. International Journal of Innovative Computing, Information and Control, 4:849-856, 2008.

[27] K. Yasuda and K. Hirai. Upper and lower bounds on the solution of the algebraic Riccati equation. IEEE Trans. Automat. Contr., 24:483-487, 1979. 